\title{
Extending HL7 RIM Model to Capture Physical Activity Data
}

\author{
Rishi Saripalle \\ School of Information Technology, Illinois State University, Normal, IL \\ rishi.saripalle@ilstu.edu
}

\begin{abstract}
Experts recommend to include physical activity in everyone's daily routine as it helps to curb various medical issues. They provide diverse healthcare services (e.g., interventions, exercise routine, personal trainer plan, therapy, etc.) to promote healthy living and to increase physical activity in one's ever increasing hectic schedules. An individual generates a lot of data when he/she performs a physical activity. However, this data (exercises, training plans, etc.) and any other associated data (e.g., results, vitals, calories, weight, etc.) is not represented using commonly agreed standards. The lack of standards has given rise to two primary issues. First, interoperability issues don't allow the physical activity data to be shared and integrated with heterogeneous healthcare systems. Second, various organizations and research programs still majorly rely on paper to record the data. This makes it highly impractical to develop any data-driven physical activity applications or evidence-based program. This article provides a detailed analysis of the problem and provides a potential solution in terms of extending HL7 RIM model using health and life sciences entities from Schema.org.
\end{abstract}

Keywords-physical activity model; exercise model; HL7 RIM physical activity; HL7 exercise; EIM; FHIR; FHIR exercise

\section{INTRODUCTION}

In general, Physical Activity (PA) is defined as any body movement that works your muscles and requires energy expenditure. It is proven that 30 minutes of daily physical activity can significantly reduce a variety of chronic diseases, improve mental health and overall well-being of an individual [1]. Even though the benefits of performing any type of physical activity on the individual's overall health has been proven, physical inactivity is still the fourth leading cause of death [2]. It also manifested in higher health care expenditures [3], [4]. To tackle physical inactivity, experts have designed numerous programs and interventions to increase physical activity among various age groups, irrespective of their medical condition [5][8]. The data obtained through these interventions/programs is recorded, analyzed and reported either using either paper (notes, journals, reports, etc.) or semi-structured digital (e.g., excel, text, etc.) format. Experts also recommend exercise - one the most common and popular way to achieve physical activity or to reach personal health goals. Exercises come in various forms and can vary from low-intensity exercises such as walking, strolling, sleeping, etc. to vigorous-intensity exercises such as running, swimming, sports, etc. Currently, most of this exercise data (e.g., the name of the exercise, repetitions, duration, process, etc.) generated by an individual/patient is recorded using paper or at best using a mobile app. For example, weight training is one the most popular exercises and individuals who include it their routine still use manuals (fitness journals or notebooks) to record their training/routine data. Further, in today's digital era, the vital sign data (heart rate, blood pressure, etc.) and other associated data (calories, step count, elevation, etc.) generated by an individual/patient during the exercise or any physical activity is recorded by wearables. The wearables constantly monitor the individual's vitals and synchronize the data to a mobile app which latter pushes the data to a data repository. Eventually, this process creates data $\operatorname{silos}^{1}$ as the data is captured by a variety of apps, represented using proprietary formats, and stored in proprietary data hubs that might not communicate with one another or to a healthcare system. In parallel, individuals also use various paper and mobile apps (e.g., MyfitnessPal ${ }^{2}$, CalorieKing ${ }^{3}$, etc.) to record their calorie consumption, food type, and nutrient information for various healthcare reasons. Similar to wearables, these mobile apps capture the data using proprietary data formats and later synchronize it to a proprietary data hub.

So, what is the problem here? First, the physical activity data captured by these various instruments (interventions or exercise training or personal training, etc.) on an individual/patient is majorly paper-based or digital format without following any standards. This gives rise to interoperability issues, an issue previously encountered with paper-based health records, that doesn't allow the data to be exchanged between heterogeneous systems, specifically with an Electronic Health Record (EHR) or any standard clinical system. What is the impact? The healthcare expert(s) do not have a wholistic view of an individual/patient health. Second, due to data interoperability issues, experts will not be able to aggregate the data from multiple diverse sources to design evidence-based physical activity programs as shown in Figure 1. For instance, Rishi Saripalle age 30 with no serious medical condition approaches a trainer to be physical activity and achieve his goals. Most of the trainers use their knowledge and experience to design an activity routine to help Rishi reach his goal. If a diet is required,

\footnotetext{
1 https://www.bedrockdata.com/blog/the-danger-of-data-silos-part-1-wheredo-they-come-from

${ }^{2}$ https://www.myfitnesspal.com/

${ }^{3}$ http://www.calorieking.com/ 
Rishi might seek assistance from a nutritionist to put a plan together that works with his training. How can the trainer and the nutritionist prove that this training and diet plan works? Currently, the physical activity domain cannot provide evidence to such questions or questions with a similar theme. However, the domain of biomedical informatics can provide evidence to such questions as its medical data can be aggregated because the data is represented and shared using healthcare standards [9].

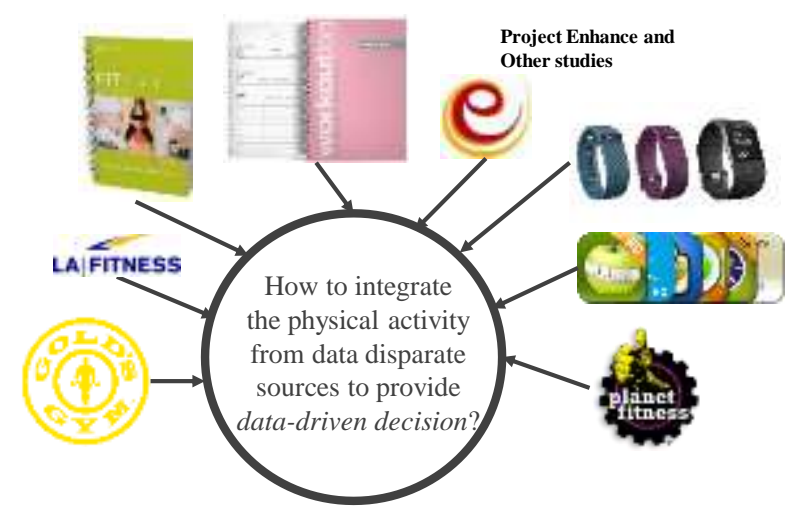

Figure 1: Interoperability issues with physical activity data.

These mentioned issues in the physical activity domain are primarily due to lack of standards, both structural and semantic, for representing and sharing physical activity data. This preliminary research is focused on structural standards. The idea is to use existing healthcare standards and other standard models/vocabulary to represent and share physical activity data across diverse healthcare systems. This goal is achieved by extending the HL7 Reference Information Model (RIM) [10] with health and life sciences entities developed by extending Schema.org model. Schema.org ${ }^{4}$ is a collaborative community effort to create and maintain schema(s) for data represented on the internet. To the best of our knowledge, this is a novel effort to capture physical activity data by extending existing healthcare standards. The rest of the article is organized as follows. Section 2 will provide the reader detailed background knowledge on HL7 RIM and Schema.org. Section 3 provides the proposed design with detailed analysis, software model(s) and its application. Section 4 concludes the article with future research.

\section{BACKGROUND INFORMATION}

The primary issue identified in the previous section is the lack of common standard(s) for representing and sharing physical activity data. The domain of biomedical and healthcare informatics faced similar crisis i.e. using paper and unstructured format for recording and sharing patient summaries, laboratory results, prescriptions, and other relevant data. The Electronic Health Record (EHR) was the viable solution and the medical community worked diligently to provide reliable and scalable standards for representing and sharing EHR. Various structural (e.g. Clinical Care Document (CCD), Clinical Document Architecture (CDA) [10], HL7 V2 and V3 messaging standards, etc.) and semantic (e.g., SNOMED-CT [11], ICD [12], etc.) standards are developed to provide a sound framework for supporting EHRs. The HL7 V2 standard was developed from the perspective of the clinicians and hospitals, but is not backed by strong software modeling principles. The HL7 V3 standard was designed using fundamental software modeling principles and formal object-oriented methodology primarily targeting biomedical informatics experts.

The HL7 Reference Information Model (RIM) is the cornerstone of the HL7 V3 messaging design and development. The RIM model has six core classes: Act, ActRelationship, Participation, Roles, RoleLinks, and Entity. Everything happening in the domain is an Act. The Act is further specialized to define other acts such as Medications, Procedures, Observations, etc. The ActRelationship connects two or more Acts using relations such as composition, precondition, revision, etc. Participation defines the context for an Act. The participants are assigned a Role such as a patient, provider, practitioner, specimen, employee, etc. Multiple roles involved in the Act are related using RoleLink. Finally, Role is assigned to Entity such as a person, organization, place, etc. In summary, HL7 V3 messaging standard is based on the HL7 Reference Information Model with six backbone classes with welldesigned hierarchies and abstraction.

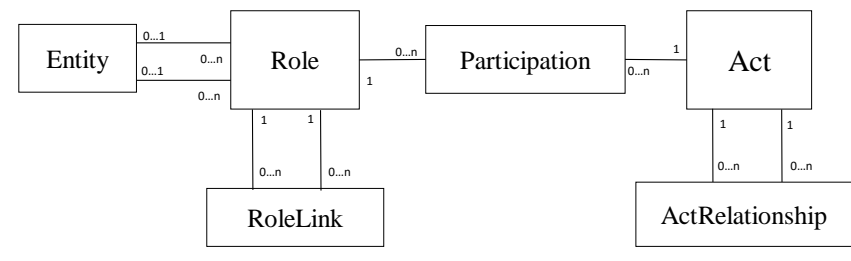

Figure 2: The HL7 Reference Information Model core classes.

Figure 3 shows a HL7 V3 message that captures "Heart Beat" (recorded as an observation (OBS)) as an outcome (OUTC) of the activity "Jogging" (also recorded as observation (OBS)).

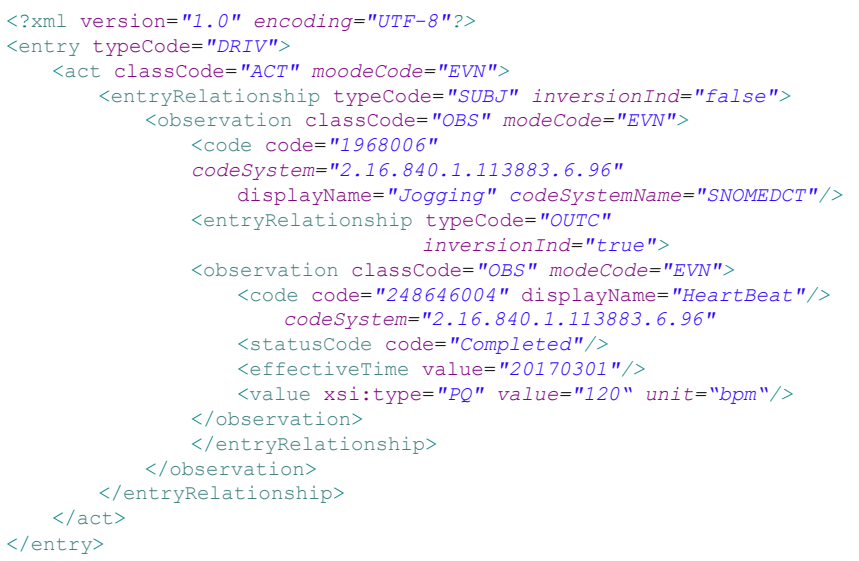

Figure 3: HL7 V3 message for representing heart beat due to jogging.

With the current standard, only a few physical activities and the associated data can be represented and shared. For example,

\footnotetext{
${ }^{4}$ http://schema.org/
} 
activities such as jogging, running, swimming, etc. and any associated data (e.g., heart rate, blood pressure, calories, etc.) generated during the activity can also be captured using RIM. However, not all physical activities can be represented using the RIM such as weight training, exercise plans, diet, therapy, etc. However, the formal object-oriented model can be extended to capture data based on the requirement - physical activity data.

To further support this statement and the research goal, the exercise community is pursuing Exercise is Medicine (EIM) [13]. The primary goal of EIM is to establish physical activity as a requirement for patients that can be prescribed by healthcare practitioners and persist it in an EHR. An EIM ${ }^{5}$ solution has three modules: Physical Activity Vital Sign (PAVS), EIM Prescription and EIM Referral. The PAVS records the physical activity levels of the patient by using the PAVS questionnaire. The EIM Prescription is the most interesting module which allows experts to prescribe a physical activity to the required patients similar to a medication prescription. Figure 4 shows the flow chart of embedding an EIM solution (three modules) into an EHR.

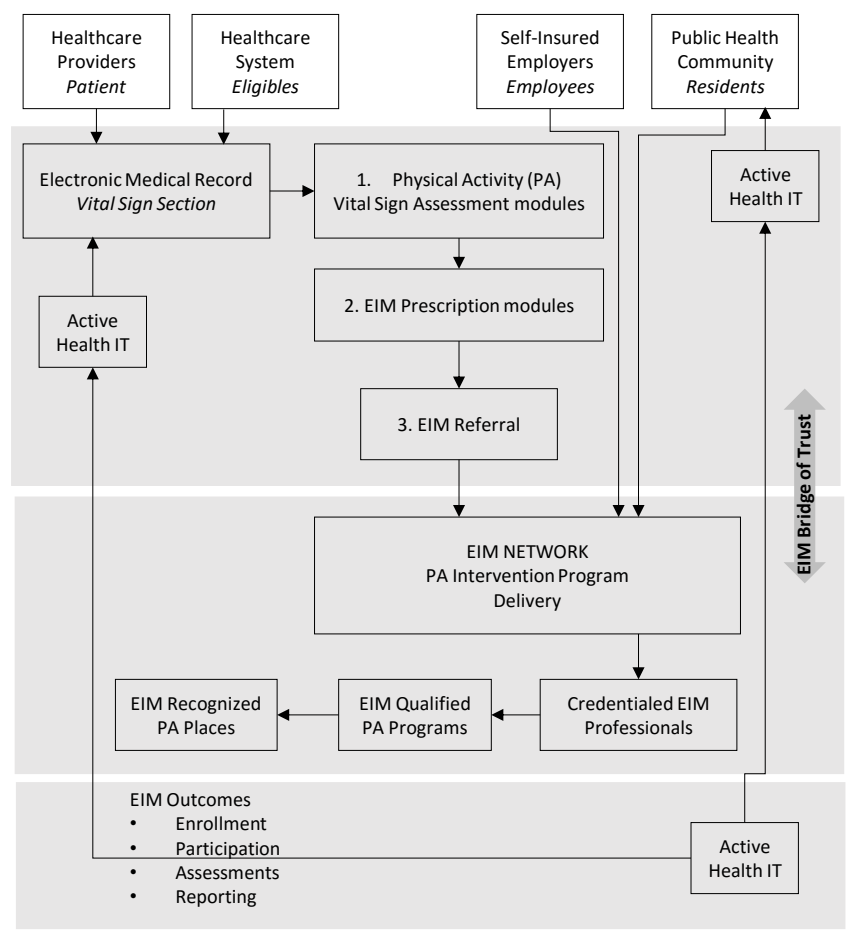

Figure 4: Exercise is Medicine in an EHR.

Apart from reusing the existing standards and community driven efforts such as EIM, there are only a handful of research studies that have identified and reported the need to capture physical activity data for persistence, longitudinal healthcare, and analysis. Kim et al., [14] have developed a physical activity semantic model that is comprised of activity keywords, qualifiers, response measures and context. These entities are obtained by reviewing 302 physical activity questions collected from standardized questionnaires and public data repositories. Sallies [15] identified the importance of physical activity similar to other experts and emphasized the need for the healthcare system to treat physical activity as a vital sign. This will manifest in recording and be observing the patient's physical levels during their medical visits. Coleman et al., [16] conducted a study to describe the facts and validity of an exercise vital sign (EVS) for use in the outpatient electronic medical records. After 18 months, $86 \%(1,537,798)$ of all eligible patients had an EVS in their electronic medical record, $36.3 \%$ of patients were completely inactive ( 0 min of exercise per week), 33.3\% were insufficiently active (more than 0 but less than 150 minutes/week), and $30.4 \%$ were sufficiently active (150 min or more per week). Lobelo et al., [17] identified that mobile health (mHealth) applications and wearables can advance the assessment and integration of physical activity (PAVS module in EIM) in clinical settings and support community-based interventions. However, none of the discussed research studies leverage or extend an existing healthcare standard which is EHR compliant to capture the physical activity data.

Schema.org is a community effort to design standard schema's to provide structure to the data published on the internet. The Schema.org vocabulary includes entities, relationships between entities, and it can be extended through the Schema.org extension model. Millions of modern sites use Schema.org to markup their webpages so that they are machine readable and search engine friendly. Figure 5 shows the description of an article using ScholarlyArticle entity on the HMTL webpage. The ScholarlyArticle is the entity (itemtype) that can be reached at http://schema.org/ScholarlyArticle and the article is described using the ScholarlyArticle attributes (itemprop).

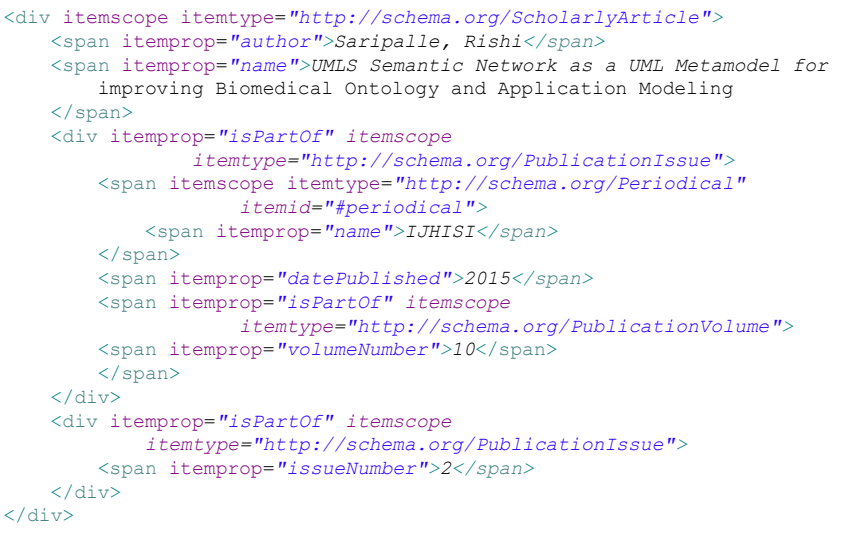

Figure 5: Representing an article using ScholarlyArticle entity in Schema.org.

This research will be using the Health and Lifesciences ${ }^{6}$, a schema designed by extending the core Schema.org vocabulary that captures medical vocabulary such as Drug, Condition, Causes, Exercise, Anatomy, Device, etc. 


\section{EXTENDING RFERENCE INFORMATION MODEL WITH ENTITIES FROM SCHEMA.ORG}

In this section will be present and discuss in detail our proposed extension to Reference Information Model (RIM) using entities from Health and Lifesciences vocabulary - an extension to core Schmea.org model. The entities that will be adapted into RIM model are: PhysicalActivity, ExercisePlan and Diet. Figure 6 shows the hierarchy from Schema.org, where ExercisePlan isa PhysicalActivity which in turn isa MedicalEntity (part of Health and Lifesciences vocabulary) which in turn isa Thing (a top concept in core Schema.org schema).

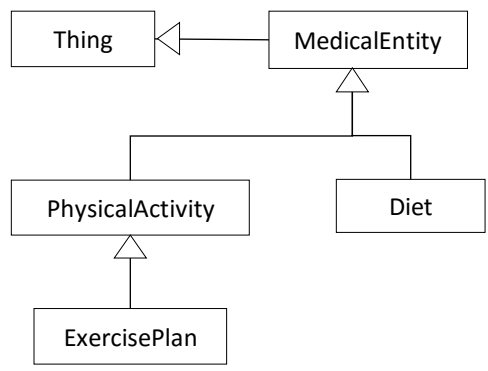

Figure 6: The hierarchical relationship between PhysicalActivity, ExercisePlan and Diet in Schema.org vocabulary.

As previously mentioned, Act in a RIM class that captures everything that happens and is extended to define other classes (Procedure, Observation, Supply, etc.). The Act is extended with the three aforementioned entities from Schema.org. It must be noted that the Diet class from RIM is merged with Diet entity from Schema.org to enrich the semantic expressiveness of the class. Figure 7 shows the extended RIM model with the Schema.org entities (in gray).

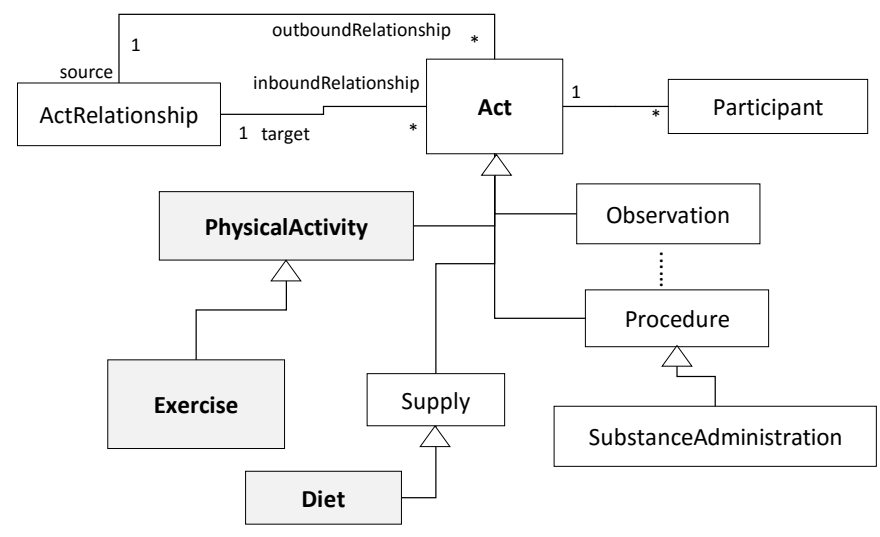

Figure 7: Extended RIM Model with Schema.org entities

As shown in Fig 7, PhysicalActivity and Exercise (renamed from ExercisePlan) are the subclasses of Act (similar to Observation) with "PHY" and "EX" as the respective classCode. The Diet entity from Schema.org is merged with the Diet class in RIM which inherits from Supply. The Schema.org entities adapted into the RIM model have their own properties and also the properties inherited from their parents, i.e. MedicalEntity and Thing (Figure 6). When these entities are defined as the subclasses of Act, they inherit the attributes and relationships of Act. Table 1 shows in detail the properties of the PhysicalActivity (with datatypes) from Schema.org and any equivalents attributes (with datatypes) PhysicalActivity class inherits from Act. Few properties of PhysicalActivity entity can be expressed as relationships with other RIM classes (Role, Entity, etc.) and a few properties can be eliminated as we are translating a Schema.org entity that is primarily used in describing data on the internet to a modeling class. The properties (expressed as "N/A" in Table 1) with no equivalent match in the Act or related to other RIM classes are defined as the attributes of PhysicalActivity class as shown in Figure 8.

TABLE 1: PhysicalActivity properties from Schema.org and equivalent attributes (relationships) in RIM model.

\begin{tabular}{|c|c|c|c|}
\hline $\begin{array}{l}\text { Property (from } \\
\text { Schema.org) }\end{array}$ & $\begin{array}{l}\text { Datatype (from } \\
\text { Schema.org) }\end{array}$ & $\begin{array}{c}\text { Equivalent } \\
\text { Attribute } \\
\text { from Act } \\
\text { Class } \\
\end{array}$ & $\begin{array}{c}\text { Equivalent } \\
\text { HL } 7 \\
\text { Datatype }\end{array}$ \\
\hline $\begin{array}{l}\text { associatedAnato } \\
\text { my }\end{array}$ & $\begin{array}{l}\text { AnatomicalStructure } \\
\text { AnatomicalSystem/ } \\
\text { SuperficialAnatomy }\end{array}$ & N/A & $\begin{array}{l}\text { CD (Coded } \\
\text { Descriptor) }\end{array}$ \\
\hline category & Text & N/A & ST (String) \\
\hline pathophysiology & Text & N/A & ST \\
\hline code & MedicalCode & Code & CD \\
\hline guideline & MedicalGuideline & N/A & ST \\
\hline alternateName & Text & N/A & $\begin{array}{c}\text { ED } \\
\text { (Encapsulat } \\
\text { ed Data) }\end{array}$ \\
\hline name & Text & Title & ED \\
\hline description & Text & Text & ED \\
\hline epidemiology & Text & $\mathrm{TX}$ & \\
\hline $\begin{array}{l}\text { Property (from } \\
\text { Schema.org) }\end{array}$ & $\begin{array}{l}\text { Datatype (from } \\
\text { Schema.org) }\end{array}$ & \multicolumn{2}{|c|}{$\begin{array}{c}\text { Expressed as a } \\
\text { relationship with a RIM } \\
\text { Class }\end{array}$} \\
\hline legalStatus & MedicalGuideline & \multicolumn{2}{|c|}{$\begin{array}{c}\text { SubstanceAdministration or } \\
\text { ST (String) }\end{array}$} \\
\hline medicineSystem & MedicineSystem & \multicolumn{2}{|c|}{ Entity } \\
\hline $\begin{array}{l}\text { recognizingAuth } \\
\text { ority }\end{array}$ & Organization & \multicolumn{2}{|c|}{ Organization } \\
\hline
\end{tabular}

The Exercise class also inherits attributes from Act as shown in Fig 7. Table 2 only shows the properties from Schema.org that will be translated as attributes in Exercise with their respective datatypes as shown in Figure 8. Table 3 shows the properties of the Diet (with respective datatypes) from Schema.org that are included in the Diet class as attributes. Figure 8 shows the complete Diet class in the extended RIM model. With the extended RIM model, the physical activity data can be shared via HL7 V3 messages. Figure 9 shows a simple HL7 V3 message that captures a new (represented using status) physical activity that affects the body composition (associatedAnatomy) of the patient and is authored by Dr. Rishi Saripalle. With availability to translate HL7 V3 to HL7 V2 messages, the message in Figure 9 can also be converted into HL V2 message, a more popular format which clinical information systems. With the extended model, an authorized organization or personal can prescribe a physical activity to a 
patient, similar to a medication and the results can be shared with the prescribed party - one of the EIM goals.

TABLE 2: Exercise properties from Schema.org and equivalent attributes datatypes in RIM model

\begin{tabular}{|c|c|c|}
\hline $\begin{array}{c}\text { Property (from } \\
\text { Schema.org) }\end{array}$ & $\begin{array}{c}\text { Datatype (from } \\
\text { Schema.org) }\end{array}$ & $\begin{array}{c}\text { Equivalent HL 7 } \\
\text { Datatype }\end{array}$ \\
\hline activityDuration & $\begin{array}{c}\text { Duration/ } \\
\text { QualitativeValue }\end{array}$ & $\begin{array}{c}\text { PQ (Physical } \\
\text { Quantity) }\end{array}$ \\
\hline activityFrequency & QualitativeValue/ Text & PQ \\
\hline additionalVariable & Text & ST \\
\hline exerciseType & Text & CD \\
\hline intensity & QualitativeValue/ Text & PQ \\
\hline alternateName & Text & ED \\
\hline repetitions & $\begin{array}{c}\text { QualitativeValue/ } \\
\text { Number }\end{array}$ & QTY (Quantity) \\
\hline restPeriods & $\begin{array}{c}\text { QualitativeValue/ } \\
\text { Number }\end{array}$ & QTY \\
\hline Workload & $\begin{array}{c}\text { QualitativeValue/ } \\
\text { Energy }\end{array}$ & PQ \\
\hline
\end{tabular}

TABLE 3: Diet properties from Schema.org and equivalent attributes

\begin{tabular}{|c|c|c|}
\hline \multicolumn{1}{|c|}{$\begin{array}{c}\text { Property (from } \\
\text { Schema.org) }\end{array}$} & $\begin{array}{c}\text { Datatype (from } \\
\text { Schema.org) }\end{array}$ & $\begin{array}{c}\text { Equivalent HL 7 } \\
\text { Datatype }\end{array}$ \\
\hline dietFeatures & Text & ST \\
\hline physiologicalBenefits & Text & ST \\
\hline risks & Text & ST \\
\hline \multicolumn{3}{|c|}{} \\
\hline $\begin{array}{c}\text { Property (from } \\
\text { Schema.org) }\end{array}$ & $\begin{array}{c}\text { Datatype (from } \\
\text { Schema.org) }\end{array}$ & $\begin{array}{c}\text { Expressed as a } \\
\text { relationship with a } \\
\text { RIM Class }\end{array}$ \\
\hline endroders & Organization/Person & Organization/Person \\
\hline
\end{tabular}

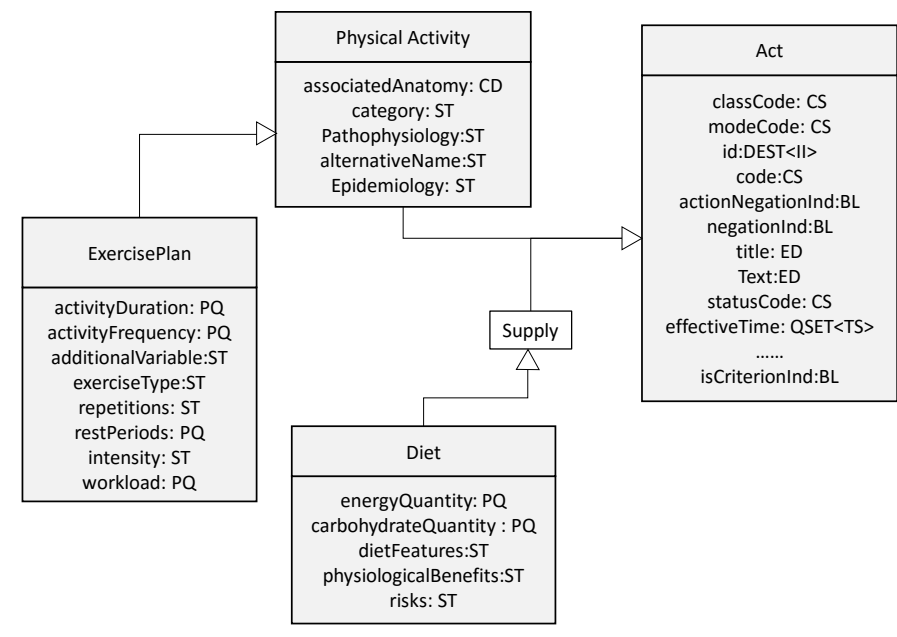

Figure 8: PhysicalActivity, Exercise and Diet classes in extended RIM model.

Figure 10 shows another HL7 message that captures a physical activity with a defined exercise plan, and an associated diet authored by an expert associated with an organization. The extended RIM model can also capture multiple exercise plans that can be associated with a physical activity. In the course of this research, the author has noticed that the physical activity/exercise/cardio domain doesn't have any standard terminology or ontology that express the vocabulary of the domain. Few existing medical standards (e.g., SNOMED) capture generic vocabulary such as jogging, walking, running, etc., but don't capture in-depth vocabulary such as weight lifting, chest press, bench press, plank, lunges, etc. The domain would require a standard vocabulary for unambiguous interpretation of shared activity data.

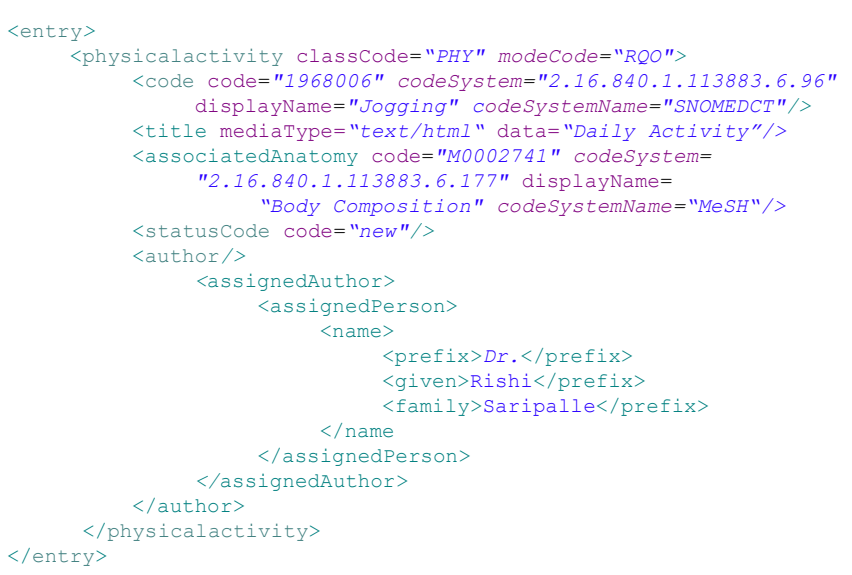

Figure 9: A HL7 V3 message capturing physical activity

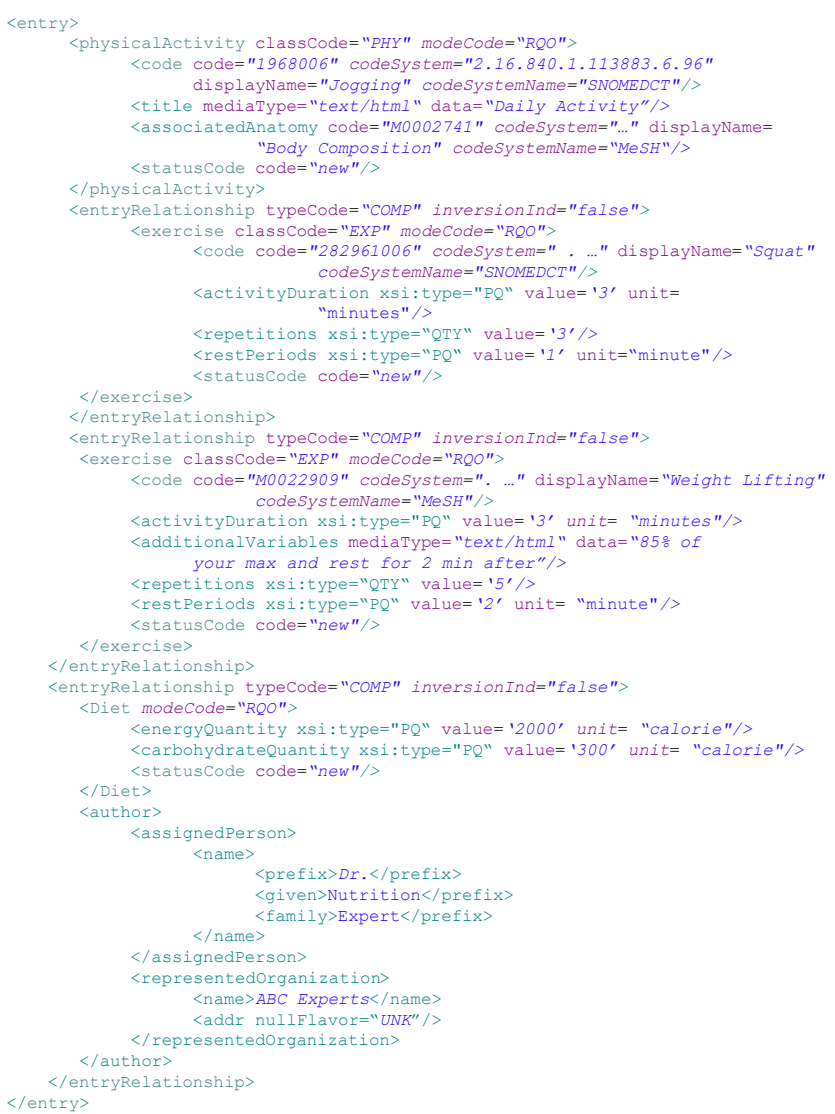

Figure 10: A HL7 V3 message capturing physical activity, an exercise plan, Diet and associated author of the Diet.

\section{CONCLUSION AND FUTURE WORK}

In this research article, existing medical standard(s) which is compliant with EHR is extended to represent and share physical activity data across heterogeneous healthcare systems. This 
goal is achieved by extending HL7 Reference Information Model (RIM) with entities defined in Health and Life Science vocabulary from Schema.org. To the best of our knowledge, this is a novel approach to represent the physical activity/exercise data using HL7 RIM and sharing it using HL7 V3/V2 messaging standards. To support this approach, in Section 1, the article presented the current situation in the domain and the immediate need for capturing the physical activity data (Fig 1). Section 2 provides the required background knowledge and other scholarly research efforts aligned with the proposed idea, especially EIM solution (Figure 4). Finally, the article demonstrates the capabilities of the extended HL7 RIM by constructing HL7 V3 messages (Fig 9 \& Fig 10) using simulated data.

The work presented here is a very crucial first step towards capturing physical activity data and moving towards evidencebased practice, similar to biomedical and healthcare informatics. However, more work is required to expand, implement and integrate the proposed model into an EHR. Apart from Schema.org entities, integrating the paper-based format followed by various fitness organizations (e.g., LA Fitness, Gold gym, etc.) and expert's knowledge would add value to the extended model. We are continuing our effort to implement the designed model using openEHR as a proof of concept. Further, with increasing use of mobile apps, ubiquitous computing for capturing data and the influence of light-weight web services, the proposed extended model classes will also be translated into Fast Healthcare Interoperability Resources $(\text { FHIR })^{7}$ resources [18]. This is achieved by using HAPI $^{8}$, an open source implementation of FHIR standard. With the integration of the physical activity data into an EHR, an expert(s) will have a wholistic view of the patient health.

\section{REFERENCES}

[1] Anonymous (2016, June 6). Physical Activity Guidelines for Americans.

[2] K. Harold et al, "The pandemic of physical inactivity: global action for public health," The Lancet, vol. 380, pp. 294, 2012.

[3] S. A. Carlson et al, "Inadequate Physical Activity and Health Care Expenditures in the United States," Prog. Cardiovasc. Dis., vol. 57, pp. 315-323, 2016/05, 2015.

[4] J. F. Bell et al, "Health-Care Expenditures of Overweight and Obese Males and Females in the Medical Expenditures Panel Survey by Age Cohort," Obesity, vol. 19, pp. 228-232, 2011.

[5] A. Carlin, M. H. Murphy and A. M. Gallagher, "Current influences and approaches to promote future physical activity in 11--13 year olds: a focus group study," BMC Public Health, vol. 15, pp. 1-12, 2015.

[6] M. Temple and J. C. Robinson, "A systematic review of interventions to promote physical activity in the preschool setting," Journal for Specialists in Pediatric Nursing, vol. 19, pp. 274-284, 2014.

[7] C. Amaya-Castellanos et al, "Development of an educational intervention to promote healthy eating and physical activity in Mexican school-age children," Eval. Program Plann., vol. 52, pp. 159, 2015.

[8] B. Belza et al, "From research to practice: EnhanceFitness, an innovative community-based senior exercise program," Topics in Geriatric Rehabilitation, vol. 26, pp. 299-309, 2010.

[9] M. Blechner, R. Saripalle and S. Demurjian, "A proposed star schema and extraction process to enhance the collection of contextual amp; semantic information for clinical research data warehouses," in 2012 IEEE International Conference on Bioinformatics and Biomedicine Workshops, 2012, pp. 798-805.

\footnotetext{
${ }^{7}$ http://hl7.org/fhir/resourcelist.html
}

[10] K. W. Boone, The $C D A^{T M}$ book. Springer, 2011.

[11] T. Benson and G. Grieve, Principles of Health Interoperability: SNOMED CT, HL7 and FHIR. Springer, 2016.

[12] (2010). International Classification of Diseases. Available: http://www.who.int/classifications/icd/en/.

[13] F. Lobelo, M. Stoutenberg and A. Hutber, "The Exercise is Medicine Global Health Initiative: a 2014 update," British Journal of Sports Medicine, vol. 48, pp. 1627, 2014.

[14] H. Kim et al, "Developing a Semantic Model to Describe Physical Activity Data," Stud. Health Technol. Inform., vol. 225, pp. 447-451, 2016.

[15] R. Sallis, "Developing healthcare systems to support exercise: exercise as the fifth vital sign," British Journal of Sports Medicine, vol. 45, pp. 473, 2011.

[16] K. J. Coleman et al, "Initial validation of an exercise "vital sign" in electronic medical records." Medicine \& Science in Sports \& Exercise, vol. 44, 2012.

[17] F. Lobelo et al, "The Wild Wild West: A Framework to Integrate mHealth Software Applications and Wearables to Support Physical Activity Assessment, Counseling and Interventions for Cardiovascular Disease Risk Reduction," Prog. Cardiovasc. Dis., vol. 58, pp. 584-594, 2016/05, 2016.

[18] M. Anwar and C. Doss, "Lighting the Mobile Information FHIR: How FHIRframe Could Dramatically Improve Mobile Health and Change HIM in the Process," Journal of AHIMA, vol. 86, 2015. 